\title{
Design of a Target Reflectance Feature Automatic Measurement System
}

\author{
Fanrong Meng ${ }^{1,2, *}$, Ning Wang ${ }^{1,2}$, Xinhong Wang ${ }^{1,2}$, Chuncheng Zhou ${ }^{1,2}$, Chuanrong $\mathrm{Li}^{1,2}$ and Lingli Tang ${ }^{1,2}$ \\ ${ }^{1}$ Key Laboratory of Quantitative Remote Sensing Information Technology, No. 9, Deng Zhuang South Road HaiDian District, \\ Beijing, China \\ ${ }^{2}$ Academy of Opto-Electronics, Chinese Academy of Sciences, No. 9, Deng Zhuang South Road HaiDian District, Beijing, China \\ ${ }^{*}$ Corresponding author
}

\begin{abstract}
A target reflectance feature automatic measurement system used for automatic radiometric calibration of satellite remote sensing payload is described in this paper. The system utilized techniques such as RS485 multi-host structure, wireless communication, and PLC. It can be used in multi-site automatic spectrum observation. The system consists of three components: the spectrum acquisition device, the data collection center and the data publishing server, covering the whole procedure from initial data acquisition to product publish. The system can realize unattended real-time spectrum measurement, obviously decreasing costs of manpower and resources relative to traditional field experiment, and can optimize the uncertainty in radiometric calibration. In addition, the system behaves good extensibility due to its adoption of RS485 multi-host structure.
\end{abstract}

Keywords-automatic measurement; GRPS; RadCalNet; reflectance feature

\section{INTRODUCTION}

On-orbit radiometric calibration and performance monitoring of remote sensing payload usually have three main ways: on-board calibration, satellite-to-satellite cross-calibration, and field (vicarious) calibration. On-board calibration uses calibration source on-board the satellite, but the performance of the source may degrade over time and can hardly provide consistent reference standard for payload testing for long periods of time. Satellite-to-satellite cross-calibration uses comparison between data required by the payload to be calibrated and data required by the synchronously overpassed reference payload, to evaluate performance of the former. But this way of calibration needs existence of high-accuracy high-stability reference payload, besides the two payloads can hardly observe ground scene in same spatial, spectral and angular properties, so the applicability of cross-calibration is limited to some extent. In recent years, plenty of field calibration experiments have been carried out throughout the world. In field calibration homogeneous ground target or natural scene is chosen as calibration site, instruments which are beforehand well-calibrated in laboratory are employed to synchronously measure surface characteristics and atmospheric condition parameters in the moment of satellite overpassing, and measured parameters will be fed into certain radiative transfer model to deduce the at-sensor incident radiance, then the radiometric calibration coefficients can be determined through comparison with the digital number value recorded by the satellite payload.
Field calibration is a feasible means to realized traceable payload calibration and product validation, but satellite-ground simultaneous observation experiment usually needs high consumption of manpower, resources and funds, and different operators and instruments used in experiment will introduce some extent uncertainties in addition.

In order to improve accuracy and consistency in field radiometric calibration and performance testing of remote sensing payload, increase observation frequency of field calibration, and better meet dynamic performance monitoring requirement for remote sensing payload, in recent years the research on automatic radiometric calibration has been more often carried out. The working group on calibration \& validation (WGCV) under the Committee on Earth Observation Satellites (CEOS) puts forward the RadCalNet (global automated radiometric calibration network) program, which object is to: choose global-located test sites capable of automatic measurement of surface and atmospheric parameters, cooperatively perform routine field automatic calibration technology research, build calibration data processing center with global consistent quality standard, promote calibration data sharing and calibration demonstration for typical satellite payloads. In this ambitious program, a key step is to develop device which can automatically measure the reflectance spectrum of ground target, however there is no mature device off-the-shelf which can finely satisfy requirements for automatic radiometric calibration. In this paper, a target reflectance feature automatic measurement system is designed and described, which is used for automatically obtaining object's reflectance spectrum characteristics and eventually serve for automatic radiometric calibration.

\section{SYSTEM DESIGN}

The target reflectance feature automatic measurement system is mainly consists of three parts: the first is a spectrum acquisition device deployed in the field, the second is a data collection center, and the third is a data publishing server. The spectrum acquisition device performs spectral data collection periodically and saves them in local storage, it also monitors data reception request from the data collection center, when capturing this request it will transfer the local data to the data collection center. The data collection center performs centralized monitoring and data collection for multiple series of spectrum acquisition devices, then sends these collected data to 
the data publishing server through the wireless module. The data publishing server receives remote data and does some appropriate processing on the spectrum data, finally publishes the data meeting quality standard into the RadCalNet website. The overall design diagram of the target reflectance feature automatic measurement system is shown in Fig I.

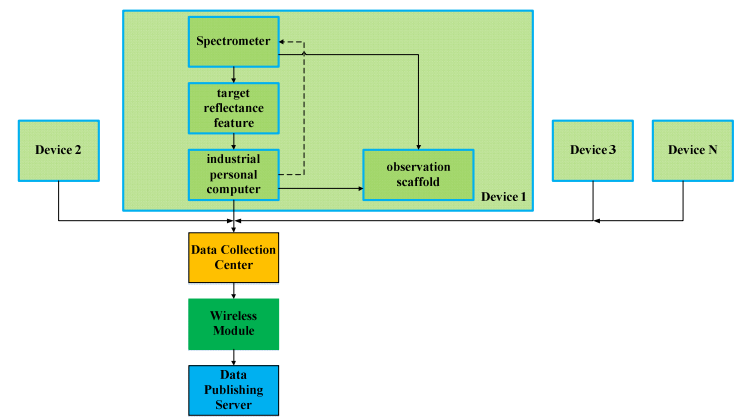

FIGURE I. OVERALL DESIGN DIAGRAM OF THE PROPOSED SYSTEM

\section{A. The Spectrum Acquisition Device}

The spectrum acquisition device is made up of observation scaffold, industrial personal computer (IPC), spectrometer and RS485 transceiver. The observation scaffold (see Fig. 2) consists of four major components: a upright pole, a cross arm, a spectrometer protection box and an IPC box. The upright pole is fixed above the ground, supporting weight of the whole device. The height of the upright pole is flexible between $1 \mathrm{~m}$ and $2 \mathrm{~m}$, can be adapted according to the site circumstances. The upright pole adopted hollow structure, inside which there is wiring channel avoiding the cable exposed to the air hence reducing its life time. The cross arm is vertical to the upright pole, with length no less than $0.5 \mathrm{~m}$, in the front of which there is a protection box for the spectrometer. A rain cap is on top of the IPC box, both sides of which have air inlet louver, and inside the IPC box there are power plugs and the temperature adjusting unit controlled by PLC. When environment temperature is higher than the upper limit, the PLC will initiate the fan; while environment temperature is lower than the lower limit, the PLC will start the heater.

The IPC is a core component of the spectrum acquisition device, which is placed inside the IPC box. The automatic measurement system device software is installed in the IPC, which is in charge of initialization of the spectrometer and PLC, control of spectrometer to acquire and save spectrum data, and response to relevant instructions from data collection center.

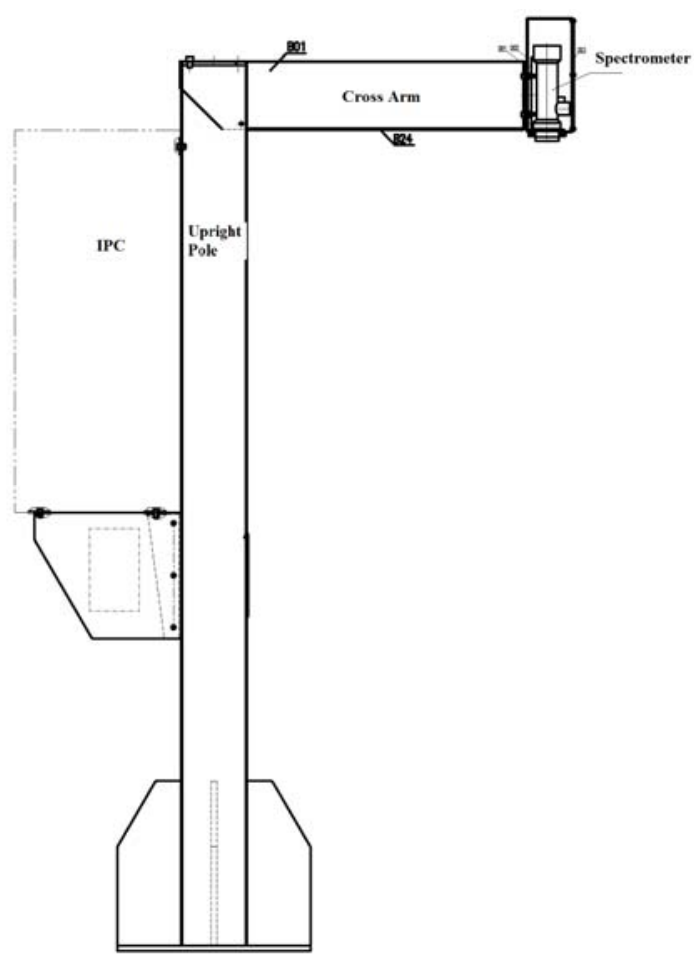

FIGURE II. STRUCTURE OF THE OBSERVATION SCAFFOLD

The spectrometer used in our system is CR280[1] spectrometer developed by Colorimetry Research Corporation, which characteristics is listed in the following table. 
TABLE I. CHARACTERISTICS OF THE CR280 SPECTROMETER

\begin{tabular}{|l|l|}
\hline Detector & $\begin{array}{l}\text { CMOS image sensor, 512 } \\
\text { pixels }\end{array}$ \\
\hline Spectral Range & 380 - $1080 \mathrm{~nm}$ \\
\hline Spectral Bandwidth & $8 \mathrm{~nm}$ \\
\hline Spectral Accuracy & \pm 0.3 nm \\
\hline Spectral Resolution & $2 \mathrm{~nm} /$ pixel \\
\hline Digital Resolution & 16 bits \\
\hline Optics & Direct viewing and direct \\
measuring system
\end{tabular}

\section{B. The Data Collection Center}

The data collection center mainly serves for spectrum data collection, and is made up of master computer, control software for the automatic measurement system, RS485 transceiver and wireless transceiver module. The data collection center is usually far away from the spectrum acquisition device, and meanwhile it is need to realize centralized control of multiple series of spectrum acquisition. Considering 485 bus has virtue of long transmission distance, strong anti-interference capability and supporting multi-master [2]communication, the RS485 transceiver is adopted to realize time-sharing control of multiple series of spectrum acquisition devices. However the spectrum acquisition device is usually placed in the field, ordinary RS485 transceiver may be easy to damage, due to complex environment (such as thunder and lightning, surge), so it is necessary to choose RS485 transceiver capable of lightning protection. In system design, we selected high performance RS485 transceiver which has transient voltage suppressor and discharge tube, providing $1500 \mathrm{~W} /$ thread lighting and surge protection, which can effectively suppress circuit surge voltage and transient over-voltage caused by lighting, ESD, etc. Tiny interpolar capacitance leads to high speed transmission ability of the RS485 interface. Baud rate is up to 128000 bps when transmission distance is within $300 \mathrm{~m}$, while up to 38400 bps when transmission distance is within $2.4 \mathrm{~km}$. The RS485 transceiver can afford working temperature of is $-25^{\circ} \mathrm{C} \sim 75^{\circ} \mathrm{C}$, relative humidity of $5 \% \sim 95 \%$, and can nicely meet field application requirement.

The control software for the automatic measurement system is installed in the master computer, which main functions include: periodically querying working condition of each acquisition device through RS485 interface, then displaying the status on the software; configuring environment temperature and working parameters of spectrum acquisition device through RS485 interface; collecting and saving data from multiple series of spectrum acquisition devices through RS485 interface by polling mode; linking with wireless transmission module through RS232 interface, in order to receive commands from the remote server data reception software, and realize data remote transmission. The display interface of the control software is shown in Fig. 3. The control software can display acquired spectral curves and acquisition device status, and is helpful for timely finding system errors and faults.

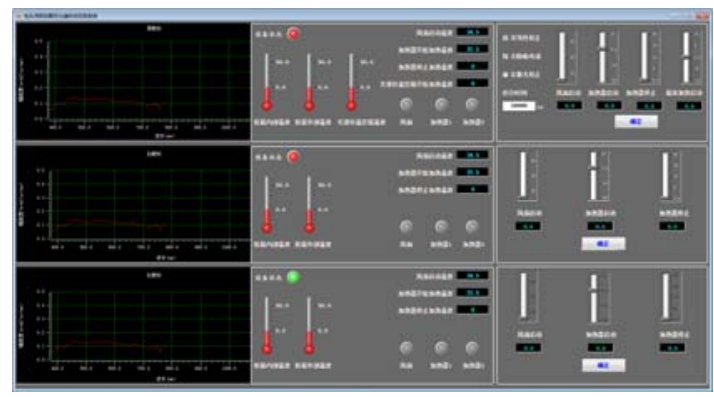

FIGURE III. INTERFACE OF THE CONTROL SOFTWARE

With development of wireless communication network, nowadays cell phone signal has realized nearly full coverage of the world. Where cable broadband network is not accessible, we can conveniently realize remote transmission of the acquired spectral data using wireless communication network. In the proposed system we choose GPRS wireless transmission module[2][3] to realize remote transmission of spectrum data. The performance of the GPRS module is listed in the following table. The module supports dual-band wireless communication, has built-in TCP/IP and UDP network protocol stack, hence is suitable for wireless transmission of spectrum data. 
TABLE II. CHARACTERISTICS OF THE GPRS MODULE

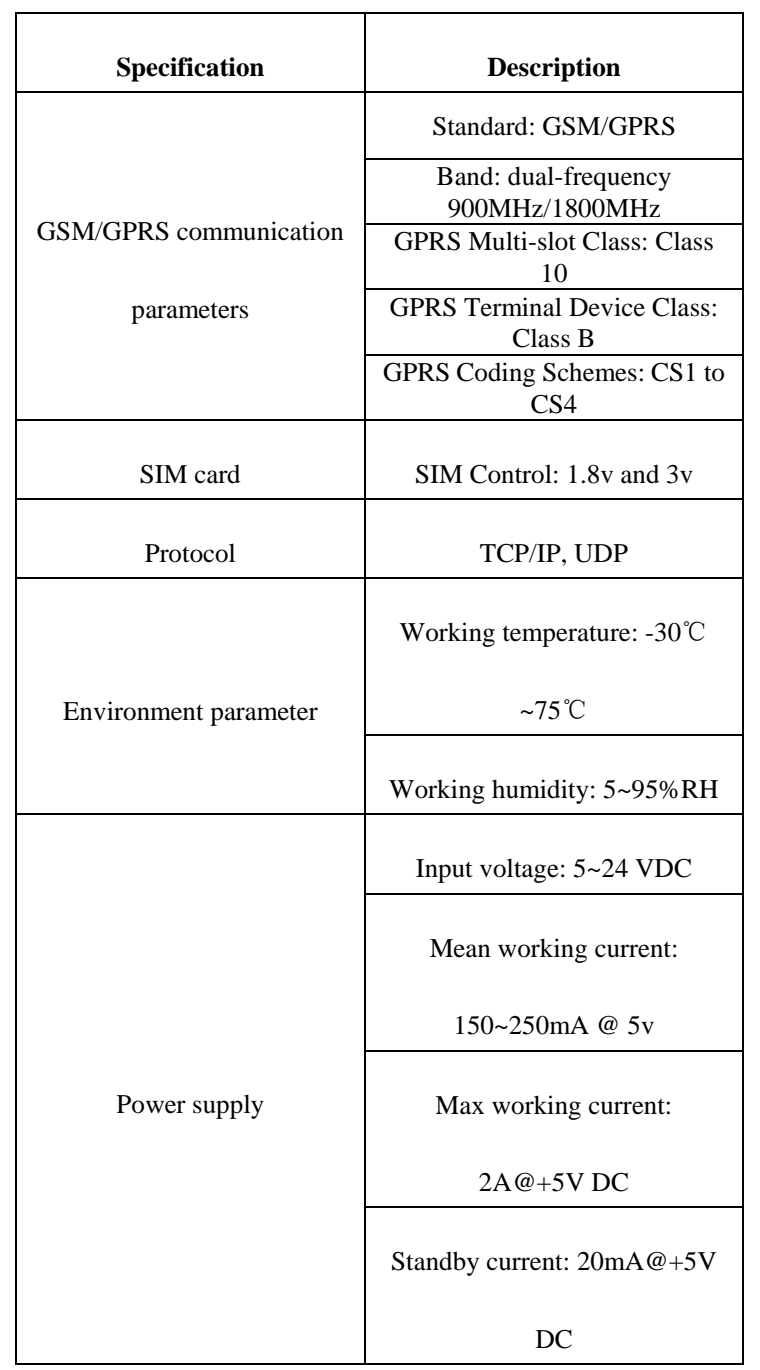

\section{The Data Publishing Server}

The data publishing server can be placed on everywhere once the network is accessible. The remote data reception software and the data post-processing software are installed in the data publishing server. The remote data reception software can communicate with the control software for the automatic measurement system through TCP/IP protocol, to realize collection and local storage of those acquired spectrum data. Received spectrum data by the remote data reception software will be input into the data post-processing software, and the output processed spectrum data will be published to the RadCalNet website, sharing to remote sensing users in the world. The interface of the remote data reception software is shown in the following Fig 4 . The real-time received spectrum data can be seen in the software, meanwhile we can judge whether the other two sub-systems are in order according to messages in the textbox window, which helps to troubleshooting.

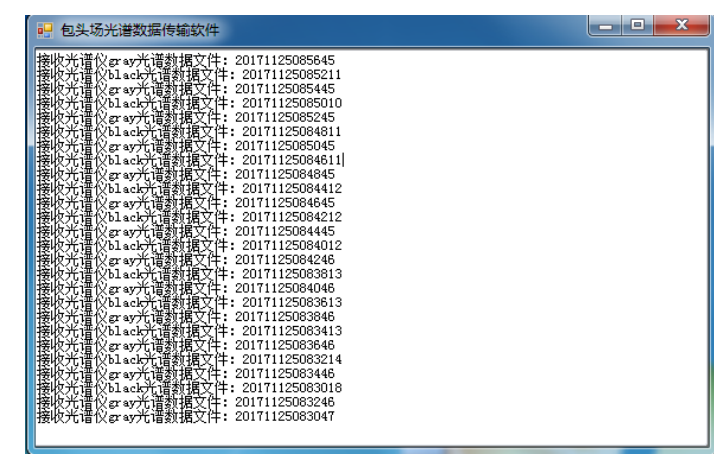

FIGURE IV. INTERFACE OF THE REMOTE DATA RECEPTION SOFTWARE

\section{Application in the Baotou Site}

The target reflectance feature automatic measurement system described above has been successfully applied in the National Calibration and Validation Site for High Resolution Remote Sensors ("the Baotou site" in brief), China. The Baotou site is one of the first-batch four demonstration sites of the RadCalNet. Three series of spectrum acquisition devices were deployed into the Baotou site. They are set up above the black permanent target, the gray permanent target and the white permanent target, respectively. The device is used to periodically acquire spectrum data of the underlying target. The data collection center locates at the office area about $500 \mathrm{~m}$ far away from the permanent target area. The data publishing server is placed in the Academy of Opto-Electronics, Chinese Academy of Sciences (locating in Beijing, China). Using the target reflectance feature automatic measurement system, obtained spectral products (standardized ground measurement spectrum data at $10 \mathrm{~nm}$ interval every 30 minutes) have been releasing to the RadCalNet website. Fig 5.(a) shows the spectrum acquisition device equipped above the white permanent target, which is been working for almost 3 years . Fig 5.(b) is an example of surface reflectance compare, the graph shows the surface reflectance represented by the green line retrieved from the auto measurement system close to the red line measured from the SVC spectrometer. 

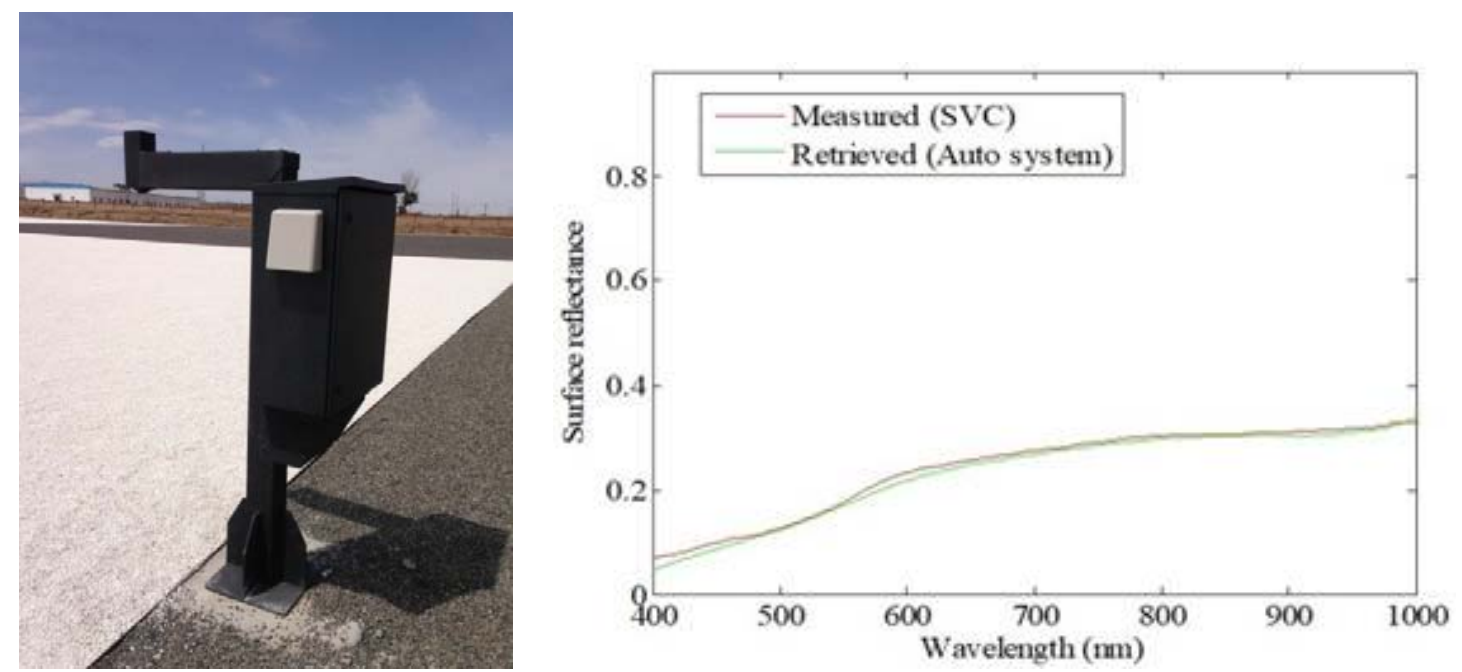

FIGURE V. (A)THE SPECTRUM ACQUISITION DEVICE ABOVE THE WHITE PERMANENT TARGET REFLECTANCE COMPARE

(B)AN EXAMPLE OF SURFACE

[5] Information on http://www.comway.com.cn/

\section{SUMMARY AND FUTURE WORK}

The target reflectance feature automatic measurement system proposed in this paper can realize automatic measurement of the surface-reflected radiance in unattended field conditions, and decrease consumption of manpower and fund in traditional field calibration experiment. The system can provide high-frequency ground target measurement data, and effectively improve the frequency of on-orbit radiometric performance monitoring for satellite payload. Using this system, the temporal consistency of measurement instrument and measured data can be maintained, thus the uncertainty of remote sensing payload radiometric calibration can be reduced to some extent. When the calibration site is far from office area and lack of power supply, we will consider the following revise to the proposed system: the data collection center sub-system may be cut off, and the spectrum acquisition device can be integrated with the wireless transceiver module, adopting solar power-supply; using wireless transceiver module, the data release server can directly communicate with the IPC in the spectrum acquisition device, to perform automatic observation.

\section{ACKNOWLEDGEMENT}

This work was financially supported by the Innovation Program of Academy of Opto-Electronics (AOE), Chinese Academy of Sciences (CAS) (Y50B06A1CY) and by the Key projects for foreign cooperation (Y60605A14Y) of Bureau of International Co-operation, Chinese Academy of Sciences.

\section{REFERENCES}

[1] Information on http://www.colorimetryresearch.com/products/cr-280

[2] Hu Ming-fei, Yang Yan, Qi Jing-qun and Ke Qi-qun, Design of Multi-master Communication

[3] Protocol Based on RS485, Automation \& Instrumentation. 30(2015) $22-24$

[4] Wei Li, Development of Remote Wireless Communication Based on GPRS and SMS, Journal of Chongqing Institute of Technology. 21(2007)124-127 